\title{
Cyclic Neutropenia and Severe Congenital Neutropenia in Patients With a Shared ELANE Mutation and Paternal Haplotype: Evidence for Phenotype Determination by Modifying Genes
}

\author{
Peter E. Newburger, MD, ${ }^{1 *}$ Talia N. Pindyck, MD, ${ }^{1}$ Zhiqing Zhu, MS, ${ }^{1}$ Audrey Anna Bolyard, RN, ${ }^{2}$ \\ Andrew A.G. Aprikyan, PhD, ${ }^{2}$ David C. Dale, $\mathrm{MD}^{2}{ }^{2}$ Gary D. Smith, $\mathrm{MD}^{3}$ and Laurence A. Boxer, $\mathrm{MD}^{4}$
}

\begin{abstract}
Background. Cyclic neutropenia $(\mathrm{CN})$ and severe congenital neutropenia $(\mathrm{SCN})$ are disorders of neutrophil production that differ markedly in disease severity. Mutations of the ELANE gene (the symbol recently replacing ELA2) are considered largely responsible for most cases of $\mathrm{CN}$ and $\mathrm{SCN}$, but specific mutations are typically associated with one or the other. Procedure. We performed ELANE genotyping on all individuals and paternal sperm in an SCN kindred with eight SCN progeny of a sperm donor and six different mothers. Results. One patient with CN had the same S97L ELANE mutation as seven patients with the SCN phenotype. The mutant allele
\end{abstract}

was detected in the donor's spermatozoa, representing $18 \%$ of the ELANE gene pool, but not in DNA from his lymphocytes, neutrophils, or buccal mucosa, indicating gonadal mosaicism. Conclusions. The coexistence of $\mathrm{CN}$ and $\mathrm{SCN}$ phenotypes in this kindred with a shared paternal haplotype strongly suggests both a role for modifying genes in determination of congenital neutropenia disease phenotypes, and the classification of $\mathrm{CN}$ and SCN within a spectrum of phenotypes expressing varying degrees of the same disease process. Pediatr Blood Cancer. 2010;55:314-317. (c) 2010 Wiley-Liss, Inc.

Key words: cyclic neutropenia; ELANE mutation; severe congenital neutropenia; sperm donor

\section{INTRODUCTION}

Cyclic neutropenia $(\mathrm{CN})$ is an autosomal dominant disorder (sporadic or inherited) characterized by regular oscillations of peripheral blood neutrophils from near normal to severely low levels, generally with 21-day periodicity. Absolute neutrophil count (ANC) nadirs are often below $200 / \mathrm{mm}^{3}$ and are associated with fever, mouth ulcers, pharyngitis, sinusitis, or more serious infections (such as colitis with gram negative sepsis) [1-3]. CN usually presents early in childhood but may run an asymptomatic course. Transformation to myelodysplastic syndrome and acute myeloid leukemia has not been reported [4,5]. Symptomatic $\mathrm{CN}$ is responsive to granulocyte colony-stimulating factor (G-CSF; filgrastim), often at low doses, which typically shortens nadir duration and increases mean and nadir ANC but usually does not ablate cycling [6,7].

Severe congenital neutropenia ( $\mathrm{SCN}$ ) is a phenotypically distinct and usually more severe disease. It is characterized by recurrent, severe infections developing in the first months of life, with ANC's consistently below $200 / \mathrm{mm}^{3}$. Bone marrow examination characteristically shows maturation arrest of neutrophil precursors at the promyelocyte/myelocyte stage of development [3,8]. Unlike CN, SCN is characterized by transformation to myelodysplasia and acute myeloid leukemia $[9,10]$. Genetic transmission may be either autosomal dominant and recessive, with many more sporadic than inherited cases reported [11-14].

$\mathrm{CN}$ and SCN appear to derive largely from heterozygous germline mutations in the ELANE (ELAstase-Neutrophil Expressed) gene, previously termed ELA2. Mutations have been found in $80-100 \%$ of $\mathrm{CN}$ cases [14-16] and in 35-63\% of SCN cases $[3,16,17]$, whether sporadic or inherited. The ELANE gene encodes neutrophil elastase, a chymotryptic serine esterase synthesized during the myeloblast to promyelocyte transition [18]. The mutant elastase likely induces accelerated apoptosis in differentiating myeloid cells $[10,19,20]$. One proposed model suggests that this accelerated apoptosis is due to the production of misfolded elastase proteins and subsequent activation of the unfolded protein response (UPR) [20,21]. However, further studies are needed to define the role of the ELANE gene and its protein product in $\mathrm{CN}$ and $\mathrm{SCN}$ pathogenesis.
While SCN and $\mathrm{CN}$ both appear to largely derive from ELANE mutations, studies have demonstrated that the type of ELANE mutation largely differs between these diseases. Specific mutations have been associated with both $\mathrm{CN}$ and SCN in unrelated patients [21], but the vast majority of mutations are associated with either $\mathrm{CN}$ or $\mathrm{SCN}$, not both. SCN and $\mathrm{CN}$-associated mutations tend to vary in genetic location and possibly, effect on elastase activity. For example, a mutation mapping study of 81 unrelated patients with $\mathrm{SCN}(\mathrm{n}=54)$ or $\mathrm{CN}(\mathrm{n}=27)$ found that $\mathrm{CN}$ ELANE mutations tend to be located in exons 2, 3, and 4 (which encode the mature polypeptide), while SCN ELANE mutations are spread over the entire five-exon sequence [16]. Although studies have suggested a relationship between individual ELANE mutations and disease severity (e.g., G185R is associated with a more severe phenotype than the R191Q mutation [21]), a more general genotype-phenotype correlation has not been established.

\section{METHODS}

All procedures and consents were approved by the human subjects committees of the investigators' institutions. Peripheral blood mononuclear cells and granulocytes were purified by Dextran sedimentation and Ficoll/Hypaque centrifugation as previously

\footnotetext{
${ }^{1}$ Department of Pediatrics, University of Massachusetts Medical School, Worcester, Massachusetts; ${ }^{2}$ Department of Medicine, University of Washington Medical School, Seattle, Washington; ${ }^{3}$ Departments of Obstetrics and Gynecology and Molecular and Integrative Physiology, University of Michigan Medical School, Ann Arbor, Michigan; ${ }^{4}$ Department of Pediatrics, University of Michigan Medical School, Ann Arbor, Michigan
}

Grant sponsor: NIH; Grant numbers: R01DK54369, R24AI049393.

Conflict of interest: Nothing to declare.

Talia N. Pindyck's present address is Internal Medicine Residency Program, Oregon Health and Science University, Portland, OR.

*Correspondence to: Peter E. Newburger, Department of Pediatrics, University of Massachusetts Medical School, Lake Avenue North, Worcester, MA 01655. E-mail: peter.newburger@umassmed.edu

Received 13 October 2009; Accepted 18 February 2010 
described. Sperm was isolated by a previously described method [22]. DNA was isolated from leukocytes, buccal swabs, and spermatozoa using the Gentra Puregene Core Kit A or QIAmp DNA Mini Kit (both from Qiagen, Inc., Valencia, CA) according to the manufacturer's recommended procedures.

PCR amplification of the affected sequence in the ELANE gene was performed with primer sets 8037F (5'-GCA CTC AAG CCA CAT CCA-3')/8706R (5'-GAT TAG CCC GTT GCA GAC-3') and 7799F (5'-TTG ATT GGC CCA CCT CAG C-3')/8706R (primer position numbers refer to the genomic sequence for ELANE, GenBank accession NG_009627). Products of PCR amplification with HotStarTaq DNA Polymerase (Qiagen, Inc.) were purified from $1 \%$ agarose gels using Qiaquick Gel Extraction Kits (Qiagen, Inc.).

The amplicons were sequenced in the forward and reverse directions using BigDye Terminator V3.1 Cycle Sequencing Kits (Applied Biosystems, Foster City, CA) and a ABI3730xl 96 capillary DNA analyzer (Applied Biosystems). Maximum peak intensities were extracted manually from raw sequencing traces using Sequence Scanner v1.0 software (Applied Biosystems). The fraction of mutant (T) versus wild-type (C) allele representation was calculated using the formula $\mathrm{T} /(\mathrm{C}+\mathrm{T}) \times 100 \%$ or $\mathrm{A} /(\mathrm{G}+\mathrm{A}) \times 100 \%$ for forward or reverse traces, respectively. Averaging of values from forward and reverse traces produced quantitative data that accurately reflected the composition of known mutant and wild-type PCR product mixtures. The fraction of mutant allele in sperm DNA was calculated from a calibration curve generated from mixtures containing $0 \%, 12.5 \%, 25 \%$, and $50 \%$ mutant alleles, with the process repeated in four independent amplification and sequencing runs.

\section{RESULTS}

\section{Patients}

A previously unreported patient was the product of conception with frozen sperm from the same donor described in our report of five SCN patients in Michigan, all progeny of the same sperm donor [13]. The current patient presented at 12 months of age with severe stomatitis and mouth ulceration. He was subsequently found to have

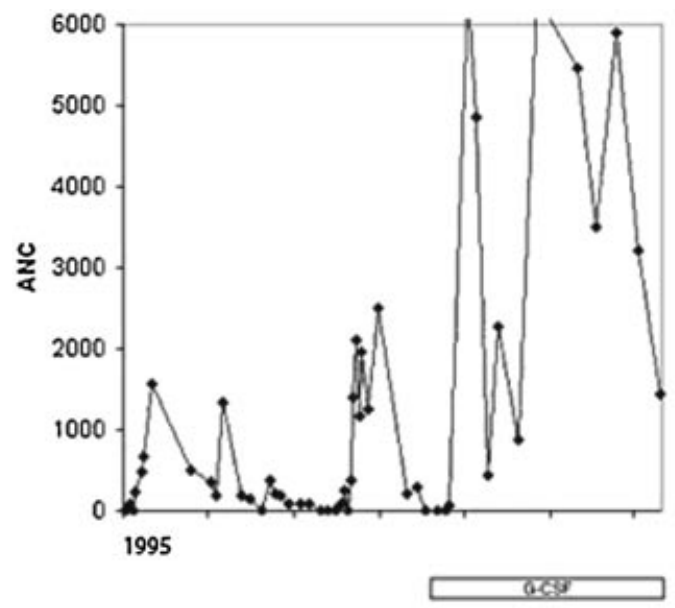

an ANC of zero and treated with antibiotics, after which his ANC began to rise. Within the following month he was readmitted for fever and neutropenia, again with stomatitis. Bi-weekly complete blood counts over the following three months showed periods of severe neutropenia (ANC $<500 / \mathrm{mm}^{3}$ ), separated by ANC peaks of 1,560 and $1,300 / \mathrm{mm}^{3}$. The pattern included two cycling episodes with 21 days or less between ANC nadirs below 200/ $\mathrm{mm}^{3}$. Figure 1 illustrates two ANC cycles identified prior to G-CSF treatment.

A bone marrow examination before G-CSF treatment showed normal cellularity with moderate myeloid hyperplasia and a leftsided myeloid series with myeloid maturation to, but not beyond, the metamyelocyte stage. An anti-neutrophil antibody test showed borderline positive signal for neutrophil-associated IgG in a flow cytometric assay. Due to his severe neutropenia and oral mucositis, the patient began G-CSF treatment and his ANC improved to values above $1,000 / \mathrm{mm}^{3}$ within 6 days. Over the following years, his ANC has continued to demonstrate cycling, with all nadirs (after the first two months of G-CSF treatment) greater than $500 / \mathrm{mm}^{3}$. In 13 cycles monitored during G-CSF therapy, 8 have shown periods of 14 days or less (also shown in Fig. 1). He currently receives treatment with $\mathrm{G}-\mathrm{CSF}$ at a dose of $1.2 \mathrm{mcg} / \mathrm{kg}$ subcutaneous daily.

Sequencing of the patient's ELANE gene showed a mutation in the fourth exon, resulting in the amino acid substitution S97L. This mutation is identical to that found to be the common cause of SCN in the previously reported progeny of the sperm donor. The affected amino acid is located on a surface loop, distant from the active site of the protease.

Further investigation has found that the donor has normal peripheral blood counts, and that his wife has borne twins with SCN. All mothers had ELANE genotyping, with no mutations identified. The resultant complete pedigree is shown in Figure 2.

\section{DNA Sequence Analysis}

The missense mutation responsible for the $\mathrm{S} 97 \mathrm{~L}$ substitution is a C8284T transition [14] in exon 4 of the ELANE gene (GenBank NG_009627). As shown in Figure 3, sequence analysis of mixtures of DNA from S97L mutant and normal peripheral blood leukocytes
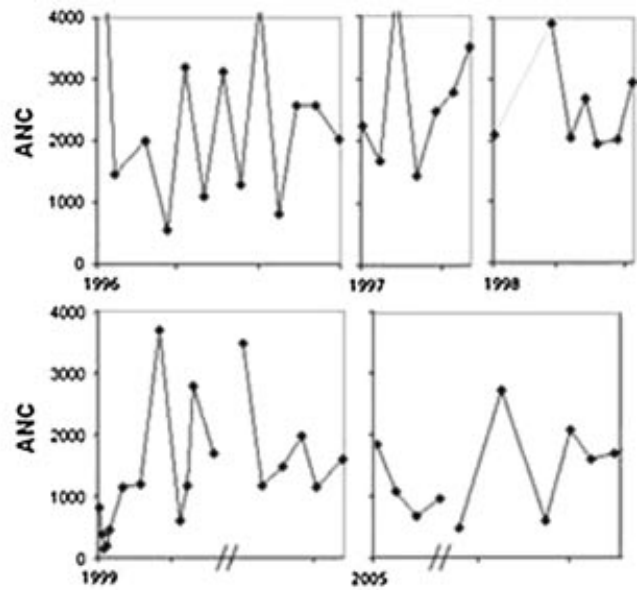

Fig. 1. CN phenotype: ANC cycling from age of 12 months to 12 years, before and during G-CSF treatment. The ordinates show ANC in units of cells $\times 10^{9} / \mathrm{L}$. Tic marks on the abscissa indicate monthly intervals. Years with no identified cycling and periods lacking ANC data are not presented. Left panel: ANCs from age 12 to 18 months in 1995, with the period of G-CSF therapy indicated by the bar in the lower margin. Right panel: ANCs in the indicated years; all during therapy with G-CSF. 


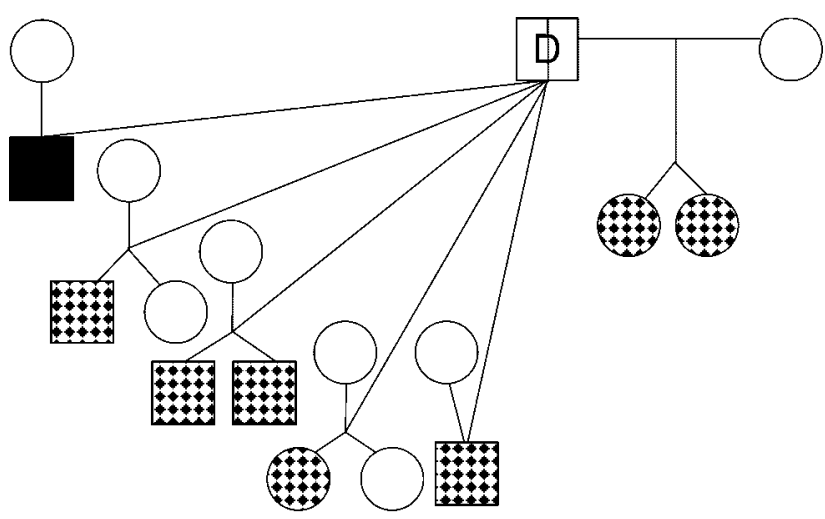

Fig. 2. Pedigree of kindred with $\mathrm{CN}$ and $\mathrm{SCN}$ phenotypes. Checkered squares (male) and circles (female) represent members with SCN. The filled square represents the patient with a $\mathrm{CN}$ phenotype. The square with a vertical dividing line and letter " $D$ " represents the sperm donor carrying the ELANE mutation. A horizontal relationship line is shown between the sperm donor and his spouse; the clustered diagonal lines indicate fertilizations by sperm donation.

showed the expected nearly equal representation of the $\mathrm{C}$ and $\mathrm{T}$ nucleotides at position 8284 (indicated by the purple box) in the mutant sample, only $\mathrm{C}$ in the normal, and a small but detectable

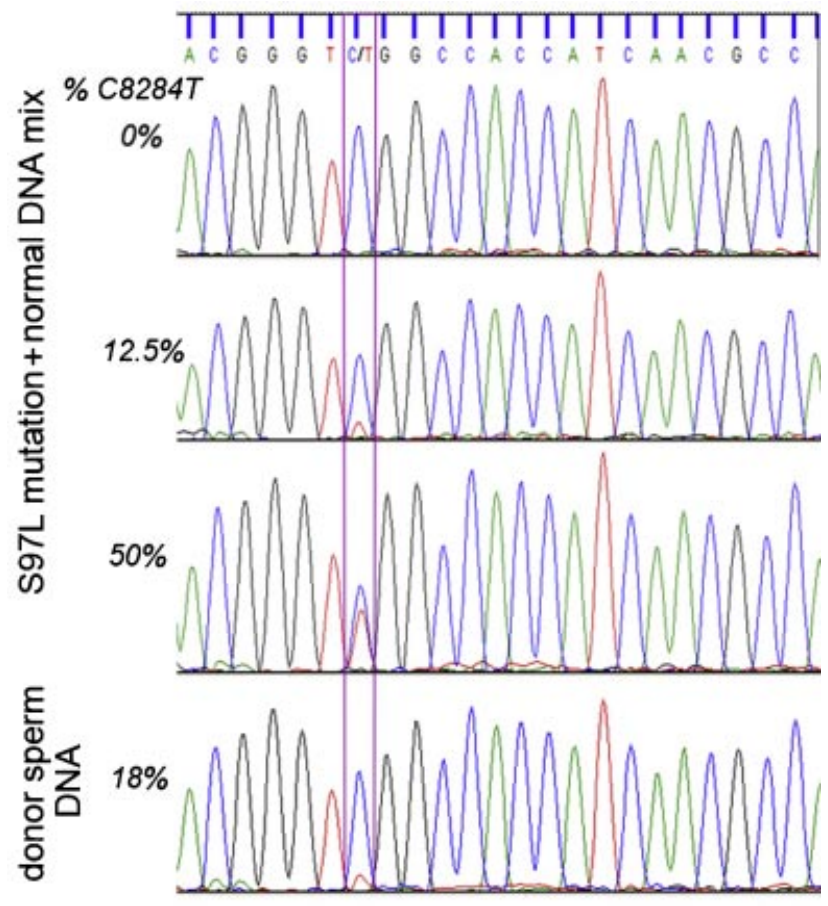

Fig. 3. DNA sequence chromograms of donor sperm and control DNA samples. The top three lanes represent mixes of $0 \%, 25 \%$, and $100 \%$ mixes of DNA from a normal donor and an SCN patient who is heterozygous for the autosomal dominant S97L mutation. The bottom lane shows sequence from the donor sperm. The numbers in italics, to the immediate left of the chromograms, indicates the calculated (for the mixes) or experimentally determined percent representation of the C8284T allele in the DNA. The purple box outlines the position of nucleotide 8284 in the sequence, which demonstrates signals for both cytosine (blue) and thymidine (red).
$\mathrm{T}$ peak in the 25:75 mutant/normal mix. The donor sperm DNA shows a similar ratio of peaks, quantitatively determined to represent $18.0 \pm 0.9 \%$ (mean $\pm \mathrm{SD}, \mathrm{n}=5$ ) mutant sequence.

\section{DISCUSSION}

This study examined a kindred that includes both a patient with a $\mathrm{CN}$ phenotype, as well as seven children with $\mathrm{SCN}$, all of whom share the same paternal haplotype. The cyclic phenotype was established by multiple documented ANCs $>500 \times 10^{9} / \mathrm{L}$ prior to G-CSF therapy, bone marrow demonstrating myeloid maturation to the metamyelocyte stage, and consistent cycling of neutrophil counts, both prior to and on G-CSF therapy. This patient also had a borderline positive anti-neutrophil antibody test, but this finding does not rule out congenital neutropenia [23]. As previously reported for five of the seven the half-siblings [13], those affected with classic SCN had consistent ANC levels $<200 \times 10^{9} / \mathrm{L}$ prior to G-CSF therapy and bone marrows showing "maturational arrest" at the promyelocyte/myelocyte stage. The current and previously reported patients all share the S97L ELANE mutation. This specific mutation has been found to be associated with multiple phenotypes [16], but not in the same family or with a shared haplotype.

The coexistence of $\mathrm{CN}$ and SCN phenotypes in this kindred strongly suggests a role for modifying genes in the determination of congenital neutropenia disease phenotypes. Recent studies report that the transcription factors, LEF-1 and Gfi-1 are linked to SCN in mice and humans [24-26]. These and other genes associated with neutrophil production may interact with a specific ELANE mutation to determine the phenotype and severity of clinical disease.

Identification of modifying genes may eventually help in the classification of patients with characteristics of both $\mathrm{CN}$ and $\mathrm{SCN}$. While $\mathrm{CN}$ and SCN are currently classified as two distinct diseases, clinical studies, and personal experiences of the authors suggest that $\mathrm{CN}$ and $\mathrm{SCN}$ represent phenotypes on a disease spectrum. For example, one of the sperm donor's progeny with SCN has demonstrated (after starting treatment with G-CSF) four consecutive ANC oscillations with magnitudes ranging from 0 to 980 and periods ranging from 16 to 24 days, as did at least one patient on the initial study demonstrating efficacy of G-CSF for treatment of SCN [27]. ANC oscillations may reflect the extent of apoptosis in differentiating myeloid cells, and thus relate to disease phenotype. Further studies of modifying genes are needed to identify these determinants, as well as to create a non-reductionist disease classification based on both genetics and phenotype [28].

The finding of $18 \%$ mutant alleles in the paternal donor's sperm explains the appearance of congenital neutropenia in eight children from six kindreds. His normal phenotype and lack of detectable mutant DNA in peripheral blood lymphocytes or buccal mucosal epithelial cells indicates that the mosaicism was likely limited to the germ cells, suggesting a new mutation during germ line development and resultant gonadal mosaicism. It is not clear whether the large number of affected offspring, from 20 or fewer fertilizations by the donor's sperm, represents a statistical anomaly or, less likely, a selective advantage for spermatozoa bearing the mutant allele.

\section{ACKNOWLEDGEMENTS}

We thank Carolyn Padden for technical assistance, Amanda Rawson for editorial assistance, and Julia Keene for preparation of the pedigree. 


\section{REFERENCES}

1. Dale DC, Hammond WP. Cyclic neutropenia: A clinical review. Blood Rev 1988;2:178-185.

2. Palmer SE, Stephens K, Dale DC. Genetics, phenotype, and natural history of autosomal dominant cyclic hematopoiesis. Am J Med Genet 1996;66:413-422.

3. Boxer LA, Newburger PE. A molecular classification of congenital neutropenia syndromes. Pediatr Blood Cancer 2007;49:609-614.

4. Donadieu J, Leblanc T, Bader MB, et al. Analysis of risk factors for myelodysplasias, leukemias and death from infection among patients with congenital neutropenia. Experience of the French Severe Chronic Neutropenia Study Group. Haematologica 2005;90:45-53.

5. Freedman MH, Bonilla MA, Fier C, et al. Myelodysplasia syndrome and acute myeloid leukemia in patients with congenital neutropenia receiving G-CSF therapy. Blood 2000;96:429-436.

6. Hammond WP, Price TH, Souza LM, et al. Treatment of cyclic neutropenia with granulocyte colony-stimulating factor. N Engl J Med 1989;320:1306-1311.

7. Dale DC, Bolyard AA, Aprikyan A. Cyclic neutropenia. Semin Hematol 2002;39:89-94.

8. Welte K, Boxer LA. Severe chronic neutropenia: Pathophysiology and therapy. Semin Hematol 1997;34:267-278.

9. Wong WY, Williams D, Slovak ML, et al. Terminal acute myelogenous leukemia in a patient with congenital agranulocytosis. Am J Hematol 1993;43:133-138.

10. Aprikyan AA, Kutyavin T, Stein S, et al. Cellular and molecular abnormalities in severe congenital neutropenia predisposing to leukemia. Exp Hematol 2003;31:372-381.

11. Ancliff PJ, Gale RE, Liesner R, et al. Mutations in the ELA2 gene encoding neutrophil elastase are present in most patients with sporadic severe congenital neutropenia but only in some patients with the familial form of the disease. Blood 2001;98:2645-2650.

12. Welte K, Dale D. Pathophysiology and treatment of severe chronic neutropenia. Ann Hematol 1996;72:158-165.

13. Boxer LA, Stein S, Buckley D, et al. Strong evidence for autosomal dominant inheritance of severe congenital neutropenia associated with ELA2 mutations. J Pediatr 2006;148:633-636.

14. Dale DC, Person RE, Bolyard AA, et al. Mutations in the gene encoding neutrophil elastase in congenital and cyclic neutropenia. Blood 2000;96:2317-2322.

15. Horwitz M, Benson KF, Person RE, et al. Mutations in ELA2, encoding neutrophil elastase, define a 21-day biological clock in cyclic haematopoiesis. Nat Genet 1999;23:433-436.
16. Bellanné-Chantelot C, Clauin S, Leblanc T, et al. Mutations in the ELA2 gene correlate with more severe expression of neutropenia: A study of 81 patients from the French Neutropenia Register. Blood 2004;103:4119-4125.

17. Rosenberg PS, Alter BP, Link DC, et al. Neutrophil elastase mutations and risk of leukaemia in severe congenital neutropenia. Br J Haematol 2007;140:210-213.

18. Papadaki HA, Eliopoulos GD. The role of apoptosis in the pathophysiology of chronic neutropenias associated with bone marrow failure. Cell Cycle 2003;2:447-451.

19. Aprikyan AA, Liles WC, Rodger E, et al. Impaired survival of bone marrow hematopoietic progenitor cells in cyclic neutropenia. Blood 2001;97:147-153.

20. Kollner I, Sodeik B, Schreek S, et al. Mutations in neutrophil elastase causing congenital neutropenia lead to cytoplasmic protein accumulation and induction of the unfolded protein response. Blood 2006;108:493-500.

21. Grenda DS, Murakami M, Ghatak J, et al. Mutations of the ELA2 gene found in patients with severe congenital neutropenia induce the unfolded protein response and cellular apoptosis. Blood 2007;110:4179-4187.

22. Sippel KC, Fraioli RE, Smith GD, et al. Frequency of somatic and germ-line mosaicism in retinoblastoma: Implications for genetic counseling. Am J Hum Genet 1998;62:610-619.

23. Boxer LA, Bolyard AA, Schwinzer B, et al. Antineutrophil antibodies lead to mistaken identity in severe congenital neutropenia. Blood (ASH Anual Meeting Abstracts) 2005;106: 385.

24. Skokowa J, Fobiwe JP, Dan L, et al. Neutrophil elastase is severely downregulated in severe congenital neutropenia $(\mathrm{CN})$ independent of ELA2 or HAX1 mutations but dependent on LEF-1. Blood 2009;114:3044-3051.

25. Person RE, Li FQ, Duan Z, et al. Mutations in proto-oncogene GFI1 cause human neutropenia and target ELA2. Nat Genet 2003;34:308-312.

26. Hochberg JC, Miron PM, Hay BN, et al. Mosaic tetraploidy and transient GFI1 mutation in a patient with severe chronic neutropenia. Pediatr Blood Cancer 2008;50:630-632.

27. Bonilla MA, Gillio AP, Ruggeiro M, et al. Effects of recombinant human granulocyte colony-stimulating factor on neutropenia in patients with congenital agranulocytosis. $\mathrm{N}$ Engl J Med 1989;320:1574-1580.

28. Loscalzo J, Kohane I, Barabasi AL. Human disease classification in the postgenomic era: A complex systems approach to human pathobiology. Mol Syst Biol 2007;3:124. 